\title{
Package Type Code
}

National Cancer Institute

\section{Source}

National Cancer Institute. Package Type Code. NCI Thesaurus. Code C93893.

A coded value specifying the kind of package. 